\title{
Термоэлектрический интенсификатор теплопередачи между двумя движущимися средами с различной температурой
}

\author{
(C) Д.К. Кадирова \\ Дагестанский государственный технический университет, \\ 367026 Махачкала, Россия \\ E-mail: kadirova.djamilya@yandex.ru
}

Поступила в Редакцию 6 марта 2019 г.

В окончательной редакции 11 марта 2019 г.

Принята к публикации 11 марта 2019 г.

Исследован запатентованный термоэлектрический интенсификатор теплопередачи между двумя движущимися средами с различной температурой. В его конструкции для увеличения интенсивности теплообмена между средами предусмотрено использование вентиляторных агрегатов. Разработана математическая модель прибора, построенная на основе решения уравнения теплового баланса по потокам сред в транспортных зонах для условий прямотока. Расчетные данные представлены в виде зависимости изменения температуры движущихся сред по длине интенсификатора при различных значениях коэффициента теплообмена между спаями термоэлектрической батареи и воздушной средой в зазоре прибора. Установлено, что чем больше разность температур сред на входе, тем более резкой будет зависимость предельной длины термоэлектрической батареи от коэффициента теплообмена.

DOI: 10.21883/FTP.2019.07.47861.41

\section{1. Введение}

В настоящее время актуальными становятся задачи исследования специальных технических средств для обеспечения интенсивной теплопередачи от источников с высокими тепловыми нагрузками к приемникам теплоты с целью выравнивания температурных уровней объектов [1-4]. Данные вопросы особенно актуальны для утилизации теплоты, выделяемой при выполнении тех или иных технологических процессов на производстве, отводе теплоты от охлаждающих жидкостей твэлов ядерных реакторов и т.п.

Одним из перспективных направлений при создании систем подобного типа является использование термоэлектрических преобразователей энергии, обеспечивающих построение экономичных, малогабаритных теплообменных аппаратов с широкими функциональными возможностями по поддержанию заданного теплового режима. Так, в этой области можно выделить работы [5-7], где исследованы возможности применения термоэлектрических преобразователей энергии для интенсификации теплообмена между потоками двух жидких или газообразных сред. Однако, несмотря на наличие теоретических и экспериментальных исследований в данной области, все еще остается актуальным вопрос по повышению интенсивности теплообмена между средами, оптимизации энергетических и массогабаритных показателей приборов.

Цель работы состоит в теоретическом исследовании термоэлектрического интенсификатора теплопередачи, в котором за счет использования принудительного продува воздушного потока вдоль спаев термоэлементов обеспечивается более высокий коэффициент теплообмена между последними и движущимися в транспортных зонах средами, температура которых подлежит изменению.

\section{2. Методика численного эксперимента}

Разработана конструкция прибора для интенсификации процесса теплопередачи между потоками двух сред, структурная схема которого изображена на рис. 1 [812]. Аппарат состоит из термоэлектрической батареи (ТЭБ) (1), составленной из идентичных по размерам и физическим свойствам термоэлементов, питаемой источником электрической энергии (на рис. 1 не показан), обе поверхности которой находятся на некотором расстоянии от стенок (2) транспортных зон (3) с движущимися в них средами (4). В начале и в конце транспортных зон (3) в направлении, перпендикулярном движению сред (4), устанавливаются вентиляторные агрегаты (5), запитываемые от того же источника электрической энергии, что и ТЭБ (1). Вентиляторные агрегаты (5) осуществляют продув воздуха в зазоре между

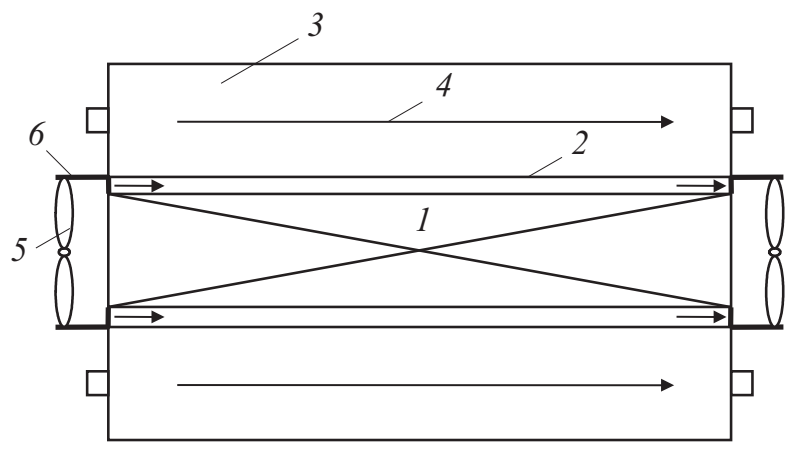

Рис. 1. Структурная схема термоэлектрического интенсификатора теплопередачи: 1 - термоэлектрическая батарея, 2 - стенка, 3 - транспортная зона, 4 - движущаяся среда (вода), 5 - вентиляторный агрегат, $6-$ крепежные приспособления. 
стенками (2) транспортных зон (3) и поверхностями ТЭБ (1), причем один вентиляторный агрегат работает на вдув воздушного потока, а второй на его выдув. ТЭБ (1), транспортные зоны (3) и вентиляторные агрегаты (5) образуют жесткую механическую конструкцию посредством крепежных приспособлений (6).

Термоэлектрический интенсификатор теплопередачи функционирует следующим образом. При пропускании через ТЭБ (1) постоянного электрического тока от источника энергии на одних спаях термоэлементов будет поглощаться теплота Пельтье, а на других — выделяться. Если холодные спаи термоэлементов будут находиться в непосредственной близости со стенкой (2) транспортной зоны (3) с горячей движущейся средой (4), а горячие спаи термоэлементов - со стенкой транспортной зоны с холодной движущейся средой, то за счет имеющегося перепада температур будет происходить интенсификация обмена тепловой энергией между двумя потоками сред. При этом продув воздуха в зазорах между стенками (2) транспортных зон (3) и поверхностями ТЭБ (1) воздушными агрегатами (5) даст возможность повысить коэффициент теплопередачи между ними за счет обеспечения режима вынужденной конвекции, при котором значение данного коэффициента выше, чем в случае кондуктивного механизма теплообмена.

Для рассмотренной конструкции разработана математическая модель, описывающая протекающие в приборе электро- и теплофизические процессы [5,13-15]. Модель построена на основе уравнений теплового баланса по потокам сред в транспортных зонах, поверхностям ТЭБ, зазорах между транспортными зонами и поверхностями ТЭБ. Рассматривается условие прямотока, подразумевающее движение потоков сред в одном направлении.

Уравнения теплового баланса в зазорах между стенками транспортных зон и спаями ТЭБ для приведенной схемы выглядят следующим образом:

$$
\begin{aligned}
& W^{\prime} \frac{d T_{1}}{d x}=\alpha^{\prime} L\left(T_{1} \text { тэБ }-T_{1}^{\prime}\right), \\
& W^{\prime} \frac{d T_{2}}{d x}=\alpha^{\prime} L\left(T_{2} \text { тЭБ }-T_{2}^{\prime}\right),
\end{aligned}
$$

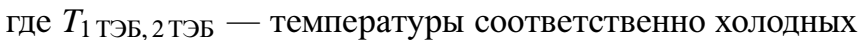
и горячих спаев ТЭБ, $T_{1,2}^{\prime}$ - температуры воздушного потока в зазорах, $W^{\prime}$ - полная теплоемкость воздушной среды, протекающей вдоль спаев ТЭБ (в зазорах) в единицу времени (равна произведению массового расхода на удельную теплоемкость среды), $L-$ длина транспортных зон, $\alpha^{\prime}$ - коэффициент теплообмена между спаями ТЭБ и воздушной средой в зазоре.

Уравнения теплового баланса по потокам сред в транспортных зонах определяются из соотношений:

$$
\begin{aligned}
& W_{1} \frac{d T_{1}}{d x}=\alpha_{1} L\left(T_{1}^{\prime}-T_{1}\right), \\
& W_{2} \frac{d T_{2}}{d x}=\alpha_{2} L\left(T_{2}^{\prime}-T_{2}\right),
\end{aligned}
$$

где $T_{1,2}-$ температуры охлаждаемых и нагреваемых сред, $W_{1}$ - полная теплоемкость среды, протекающей вдоль холодных спаев ТЭБ в единицу времени, $W_{2}-$ полная теплоемкость среды, протекающей вдоль горячих спаев ТЭБ в единицу времени, $\alpha^{\prime}-$ коэффициент теплообмена между охлаждаемой воздушной средой в зазоре и охлаждаемой средой в транспортной зоне, $\alpha_{1}$ - коэффициент теплообмена между нагреваемой воздушной средой в зазоре и нагреваемой средой в транспортной зоне.

Уравнения теплового баланса на спаях ТЭБ со стороны термоэлементов имеют вид

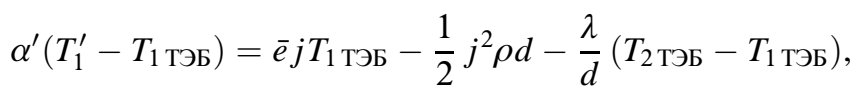

$\alpha^{\prime}\left(T_{2 \text { ТЭБ }}-T_{2}^{\prime}\right)=\bar{e} j T_{2 \text { тЭБ }}+\frac{1}{2} j^{2} \rho d-\frac{\lambda}{d}\left(T_{2 \text { ТЭБ }}-T_{1 \text { ТэБ }}\right)$,

где $\bar{e}-$ коэффициент термоэдс термоэлементов, $j$ - плотность электрического тока, $\rho-$ удельное электрическое сопротивление ветвей термоэлементов, $\lambda$ - удельный коэффициент теплопроводности ветвей термоэлемента, $L$ - высота ветвей термоэлементов.

Задача была одномерной, решение системы уравнений (1)-(6) произведено методом конечных элементов.

\section{3. Результаты численного эксперимента}

Результаты расчетов представлены на рис. 2. В качестве сред выступала вода, характеристики термоэлементов следующие: $\lambda=1.5 \mathrm{BT} /(\mathrm{M} \cdot \mathrm{K}), \quad \rho=$ $=10.7 \cdot 10^{-6} \mathrm{OM} \cdot \mathrm{M}, \bar{e}=0.2 \cdot 10^{-3} \mathrm{~B} / \mathrm{K}, d=0.003 \mathrm{M}$. Коэффициенты теплообмена: $\alpha_{1}=\alpha_{2}=100 \mathrm{BT} /\left(\mathrm{м}^{2} \cdot \mathrm{K}\right)$, величины $W^{\prime}=90 \mathrm{BT} / \mathrm{K}, W_{1}=W_{2}=120 \mathrm{BT} / \mathrm{K}$.

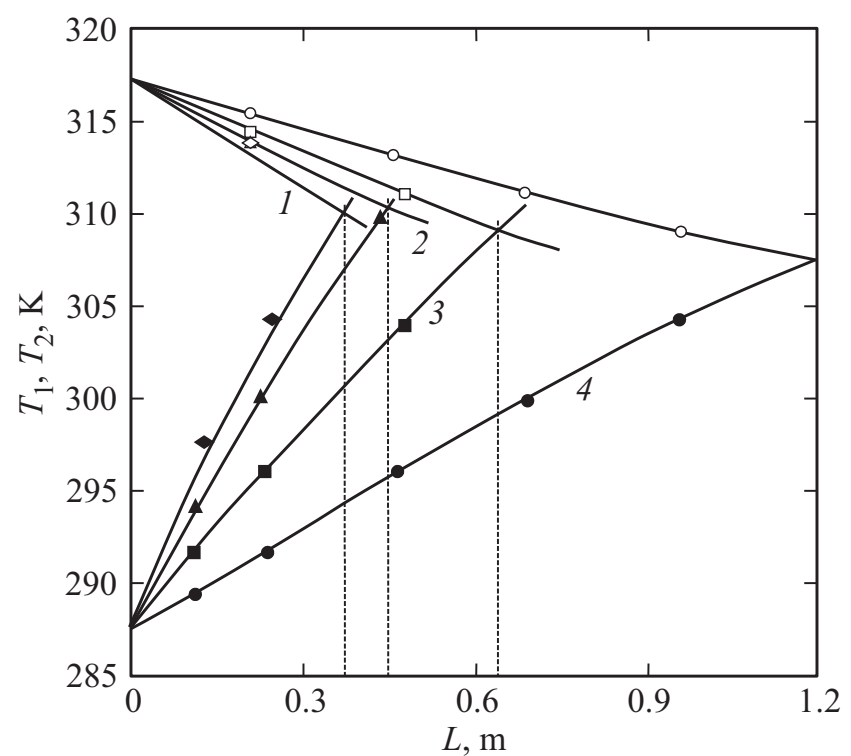

Рис. 2. Изменение температуры сред на выходе из интенсификатора теплопередачи в зависимости от его длины: $1-\alpha^{\prime}=90 \mathrm{BT} /\left(\mathrm{M}^{2} \cdot \mathrm{K}\right), 2-\alpha^{\prime}=80 \mathrm{BT} /\left(\mathrm{M}^{2} \cdot \mathrm{K}\right)$, $3-\alpha^{\prime}=70 \mathrm{BT} /\left(\mathrm{m}^{2} \cdot \mathrm{K}\right), 4-\alpha^{\prime}=60 \mathrm{BT} /\left(\mathrm{m}^{2} \cdot \mathrm{K}\right) ; I=5 \mathrm{~A}$. 


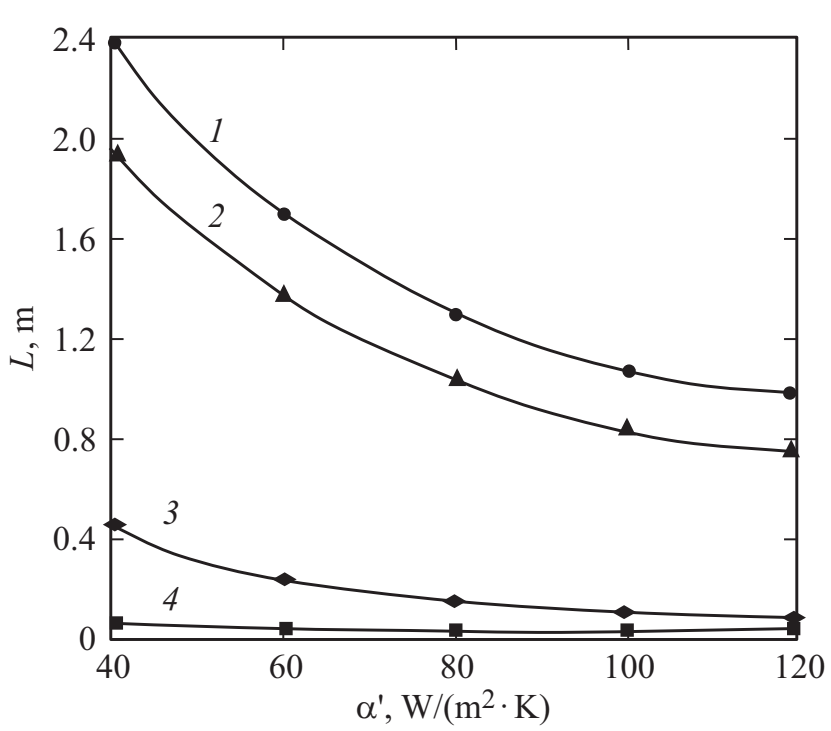

Рис. 3. Зависимости предельных длин ТЭБ в зависимости от значения $\alpha^{\prime}: 1-\Delta T=40 \mathrm{~K}, 2-T=30 \mathrm{~K}, 3-T=10 \mathrm{~K}$, $4-T=5 \mathrm{~K} ; I=5 \mathrm{~A}$.

Полученные изменения температуры сред на выходе из интенсификатора теплопередачи в зависимости от его длины при фиксированной величине тока питания ТЭБ, равной $5 \mathrm{~A}$, представлены на рис. 2. Согласно полученным данным, увеличение значения $\alpha^{\prime}$ дает возможность снизить температуру в транспортной зоне с горячей движущей средой, или увеличить температуру в транспортной зоне с холодной движущей средой, что позволяет снизить разность температур сред на выходе из теплообменного аппарата при его одинаковой длине. Так, изменение $\alpha^{\prime}$ на $10 \mathrm{BT} /\left(\mathrm{m}^{2} \cdot \mathrm{K}\right)$ в среднем изменяет температуру охлаждаемой среды на $2 \mathrm{~K}$, а нагреваемой на $3 \mathrm{~K}$.

На рис. 3 представлены графики изменения предельных длин ТЭБ (длин, при которых температуры жидкостей на выходе из интенсификатора теплопередачи равны между собой) в зависимости от значения $\alpha^{\prime}$. Как следует из представленных данных, чем больше разница температур теплоносителей на входе в устройство, тем больше длина ТЭБ, необходимая для удержания режима интенсификации, т. е. сохранения нужного режима работы. Графики носят монотонно убывающий характер в зависимости от коэффициента теплообмена между спаями ТЭБ и воздушной средой в зазоре. Чем больше разница температур сред на входе, тем резче убывают функции $L=L\left(\alpha^{\prime}\right)$ при постоянном токе питания $I=5 \mathrm{~A}$.

\section{4. Заключение}

Предложена конструкция термоэлектрического интенсификатора теплопередачи, в которой для увеличения коэффициента теплообмена между спаями термоэлементов и движущимися в транспортных зонах средами использован принудительный продув воздушного потока, максимальная скорость которого $0.0034 \mathrm{~m}^{3} / \mathrm{c}$, который дает возможность повысить коэффициент теплопередачи между транспортными зонами за счет обеспечения режима вынужденной конвекции.

Разработана модель термоэлектрического интенсификатора теплопередачи, построенная на основе решения уравнений теплового баланса по потокам сред в транспортных зонах, поверхностям ТЭБ, зазорах между транспортными зонами и поверхностями ТЭБ для условий прямотока.

Совокупность результатов проведенных исследований позволяет использовать их в качестве научной основы в дальнейшем при разработке и создании термоэлектрических интенсификаторов повышенной эффективности.

Установлено, что чем больше разность температур сред на входе, тем более резкой будет зависимость предельной длины ТЭБ от коэффициента теплообмена.

\section{Список литературы}

[1] Т.А. Исмаилов. Термоэлектрические полупроводниковые устройства и интенсификаторы теплопередачи (СПб., Политехника, 2005).

[2] Л.И. Анатычук. Термоэлементы и термоэлектрические устройства (Киев, Наук. думка, 1979).

[3] Л.П. Булат. Холодильная техника, 7, 34 (2009).

[4] Г.А. Дрейцер, И.Е. Лобанов. ИФЖ, 76, 46 (2003).

[5] И.А. Попов, Х.М. Махянов, В.М. Гуреев. Физические основы и промышленное применение интенсификации теплообмена (Казань, Логос, 2012).

[6] Ю.Ф. Гортышов, В.В. Олимпиев, И.А. Попов. Изв. РАН. Энергетика, 3, 102 (2002).

[7] G. Walker. Industrial Heat Exchangers (Washington, Hemisphere Publishing, 1990).

[8] G.F. Hewitt. Heat Exchanger Design Handbook (Begell House, 1990).

[9] Т.А. Исмаилов, О.В. Евдулов, Д.К. Кадирова, Д.В. Евдулов, Р.Ш. Казумов. Патент РФ № 2651096 (2018).

[10] M. Sajid, I. Hassan, A. Rahman. Energy, 118, 1035 (2017).

[11] A. El-Desouky, M. Carter, A.A. Matthieu, P.M. Bardet. S. Progress in Mater. Sci., 83, 330 (2016).

[12] Ch. Gaynera, K.K. Kara. Appl. Energy, 168, 65 (2016).

[13] T. Zhang. Renewable and Sustainable Energy Reviews, 38, 903 (2014).

[14] E.Virjogheb, D. Enescua. Appl. Thermal Engin., 66, 15 (2014).

[15] S.B. Riffat, M. Xiaoli. Appl. Thermal Engin., 64, 252 (2014).

Редактор Г.А. Оганесян 
Thermoelectric intensifier of heat transfer between two moving media with different temperatures

\section{D.K. Kadirova}

Daghestan State Technical University, 367026 Makhachkala, Russia

Abstract The patented thermoelectric intensifier of heat transfer between two moving media with different temperatures was investigated. In its design, to increase the intensity of heat exchange between media, the use of fan units is provided. A mathematical model of the device was developed, based on the solution of the heat balance equation for media flows in transport zones for forward flow conditions. The calculated data are presented in the form of the dependence of the temperature change of moving media along the length of the intensifier for different values of the heat transfer coefficient between the junctions of the thermoelectric battery and the air medium in the gap of the device. It was established that the larger the temperature difference between the inlet media, the more abrupt the dependence of the thermopile limit on the heat transfer coefficient will be.

Публикация материалов Конференции завершена. 